\title{
Author Correction: Thermally activated delayed fluorescence (TADF) organic molecules for efficient $X$-ray scintillation and imaging
}

Wenbo Ma (D), Yirong Su, Qisheng Zhang, Chao Deng, Luca Pasquali (D), Wenjuan Zhu, Yue Tian (D), Peng Ran, Zeng Chen, Gaoyuan Yang, Guijie Liang, Tianyu Liu, Haiming Zhu (D), Peng Huang, Haizheng Zhong (D), Kangwei Wang, Shaoqian Peng, Jianlong Xia, Huafeng Liu, Xu Liu and Yang Michael Yang (D)

Correction to: Nature Materials https://doi.org/10.1038/s41563-021-01132-x, published online 11 November 2021.

In the version of this article initially published, the scale bars in Fig. 4, panels b, e and g, were sized incorrectly and are now replaced, while the Fig. 4 e,g scale bars were mislabeled (" $2 \mathrm{~mm}$ " and " $5 \mathrm{~mm}$ " are changed to now read " $5 \mathrm{~mm}$ " and " $1 \mathrm{~cm}$," respectively). The errors affected the presentation of the figure, but do not impact the conclusions in the paper. The changes have been made in the HTML and PDF versions of the article.

Published online: 4 March 2022

https://doi.org/10.1038/s41563-022-01226-0

(c) The Author(s), under exclusive licence to Springer Nature Limited 2022 